\title{
Trombólise em Acidente Vascular Isquêmico Cardioembólico
}

\author{
Thrombolysis in Cardioembolic Stroke
}

\author{
Gisele Sampaio Silva ${ }^{1}$
}

\section{SUMMARY}

Cardioembolic stroke accounts for approximately $15 \%$ of all strokes and is thought to be one of the more preventable types of stroke. Features that have been reported to support cardioembolism as a mechanism for ischemic stroke include documented cardiac source of embolism, maximal neurologic deficit at onset, involvement of multiple cerebrovascular territories, enhanced tendency toward hemorrhagic transformation, enhanced risk of syncope or seizure at presentation, and lower likelihood of premonitory transient ischemic attacks. Since the approval of intravenous recombinant tissue plasminogen activator (rt-PA) for the treatment of acute ischemic stroke within 3 hours of stroke onset, this therapy has been used as the only proved medication capable of diminishing disability in this often devastating disease. This article reviews the peculiarities of thrombolysis in cardioembolic strokes.

\section{Keywords: Stroke, Thrombolytic Therapy.}

Citation: Silva GS. Thrombolysis in Cardioembolic Stroke. Ver Neurocienc 2006; 14(4):214-219.

\section{RESUMO}

Acidentes vasculares cerebrais isquêmicos (AVCi) cardioembólicos são responsáveis por aproximadamente 15\% de todos os AVCi. Algumas características são sugestivas de cardiembolia como mecanismo do AVCi, dentre elas: déficit máximo no início dos sintomas, múltiplos territórios vasculares acometidos, uma maior tendência à transformação hemorrágica, maior risco de crises epilépticas ou síncope no início dos sintomas e menor chance de ataques isquêmicos transitórios (AIT) premonitórios. Desde a aprovação do ativador do plasminogênio tecidual recombinante (rt-PA) para o tratamento do AVCi quando administrado até três horas do início dos sintomas, essa terapêutica tem sido utilizada como a única capaz de prevenir incapacidade nessa doença por vezes devastadora. Esse artigo faz uma revisão das peculiaridades do tratamento trombolítico no AVCi cardioembólico.

\section{Unitermos: Acidente cerebrovascular, Terapia Trombolítica.}

Citação: Silva GS. Trombólise em Acidente Vascular Isquêmico Cardioembólico. Rev Neurocienc 2006; 14(4):214-219.

\section{Embolia cerebral: uma longa história}

O conceito de que eventos trombóticos em artérias nutridoras podem levar a infartos cardíacos, renais e de outros órgãos se desenvolveu no meio do século XIX. Já em 1453, Versalius descreveu depósitos não naturais no átrio esquerdo de um paciente com gangrena na perna' ${ }^{1}$. O patologista alemão Rudolph Virchow merece o maior crédito pela descrição de danos a órgãos secundários à oclusão vascular, além de descrever que o trombo poderia se formar localmente (trombose) ou à distância (embolia)². William Osler menciona, em várias edições de seu livro texto de medicina, embolia como causa de amolecimento do tecido cerebral; no entanto, admite apenas a estenose mitral e a endocardite como prováveis causas embólicas 3 . Apenas em 1950, após as descrições por Miller Fisher das características clínicas e patológicas da trombose carotídea, é que os aspectos clínicos da doença cerebrovascular foram reconhecidos e difundidos ${ }^{4}$.

\section{Patologia, Fisiopatologia e Localização da Embolia Cerebral}

Ao contrário de trombos, que são formados localmente em áreas com aterosclerose ou outros danos arteriais, êmbolos são fracamente aderentes às paredes 
vasculares. A oclusão súbita de uma artéria intracraniana por um êmbolo leva a déficits por disfunção da região cerebral que deixou de ser suprida. Quando circulação colateral se desenvolve, os sintomas podem se estabilizar ou até melhorar. As partículas não aderentes geralmente não permanecem no local da obstrução inicial e se movem à distância. Arteriografias seqüenciais ou mesmo injeções de contraste no mesmo exame de arteriografia podem identificar a passagem do êmbolo e a saída do local de origem inicial. O deslocamento do êmbolo pode causar novos sintomas por alojamento distal em ramo arterial ou ser assintomático ${ }^{5,6}$.

A transformação hemorrágica é mais freqüente em infartos embólicos. A obstrução de uma artéria intracraniana causa dano não só ao tecido cerebral, mas também aos vasos sangüíneos da área isquêmica. Quando o êmbolo responsável pela oclusão migra distalmente, as arteríolas e capilares que sofreram isquemia permitem o extravasamento de sangue para o tecido infartado ${ }^{6,7}$. Dissecção proximal em tronco da artéria cerebral média (ACM) já foi descrita como causa de transformação hemorrágica maciça em paciente idosa com AVCi cardioembólico ${ }^{8}$.

Cerca de $80 \%$ das embolias cerebrais originárias do coração se dirigem à circulação anterior. Os restantes 20\% acometem a circulação posterior, uma proporção compatível com o débito cardíaco destinado às duas circulações. A artéria receptora do êmbolo depende do tamanho e da natureza das partículas. Partículas cálcicas, originadas em válvulas cardíacas, são menos móveis e se adaptam pior ao formato das artérias receptoras do que trombos vermelhos (ricos em fibrina e eritrócitos) ${ }^{9}$.

Embolia para a ACM pode levar a uma variedade de padrões de infarto. Oclusões do tronco da ACM antes das artérias lenticuloestriadas podem levar a infarto de todo o território dessa artéria, incluindo núcleos da base, cápsula interna e córtex cerebral. Em alguns pacientes, quando o tronco da ACM é ocluído, desenvolve-se uma circulação colateral para a convexidade cerebral, sendo poupado o território superficial da ACM. O infarto resultante é, então, limitado aos núcleos da base e à substância branca adjacente, e é denominado infarto estriato-capsular. Embolia para a divisão superior da ACM leva a infarto córtico-subcortical da convexidade supra-silviana, enquanto embolia para a divisão inferior resulta em infarto limitado ao lobo parietal, abaixo da fissura silviana. Êmbolos pequenos podem causar infartos córtico-subcorticais distais envolvendo um ou mais giros ${ }^{10,11}$ (Figura 1).

Êmbolos para a circulação posterior podem ocluir as artérias vertebrais cervicais ou intracranianas. Êmbolos que ultrapassam as artérias vertebrais intracranianas geralmente ocluem a artéria basilar distal (topo da basilar) ou um de seus ramos ${ }^{12}$.

\section{Manifestações Clínicas do AVCi Cardioembólico}

Apesar de não serem sensíveis ou específicas para

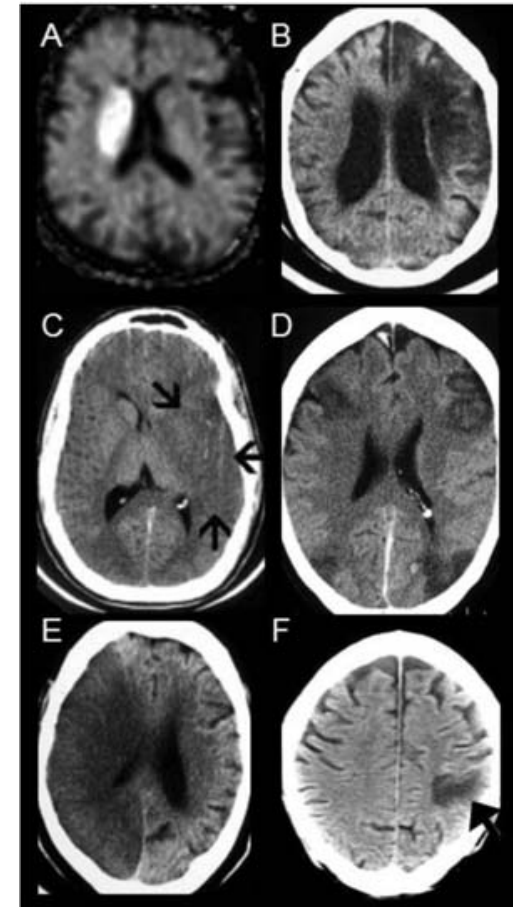

Figura 1:

$1 A$ - Ressonância magnética (seqüência de difusão) de crânio em paciente com fibrilação atrial mostrando infarto agudo em território lenticuloestriado.

1B - Tomografia computadorizada de crânio mostrando infarto em território da divisão superior da artéria cerebral média e cerebral anterior, em pacientes portadora de válvula cardíaca metálica. 1C - Tomografia computadorizada de crânio mostrando infarto em todo território da artéria cerebral média esquerda em paciente com trombo em ventrículo esquerdo.

1D - Tomografia computadorizada de crânio mostrando múltiplos infartos corticais em paciente com estenose mitral secundária à febre reumática e fibrilação atrial.

$1 E$ - Tomografia computadorizada de crânio mostrando infarto hemisférico à direita em paciente com endocardite bacteriana de válvula mitral.

1F - Tomografia computadorizada de crânio mostrando pequeno infarto cortical em paciente com forame oval patente, aneurisma de septo inter-atrial e trombose de membros inferiores.

estabelecer o diagnóstico de cardioembolia, algumas características clínicas são descritas com uma maior freqüência em AVCis cardioembólicos ${ }^{13,14,15}$ :

- Rebaixamento do nível de consciência no início dos sintomas. O tamanho maior do êmbolo de origem cardíaca é a explicação mais provável para esse achado clínico.

- Início súbito dos sintomas, com maior intensidade no início do quadro clínico.

- Recuperação rápida dos sintomas por migração do êmbolo ou reperfusão espontânea ("spetacular shrinking deficit")

- Envolvimento de mais de um território vascular cerebral.

- Déficits corticais (afasia, defeitos de campo visual). 


\section{Epidemiologia do AVCi Cardioembólico}

Aproximadamente $20 \%$ dos AVCi nos Estados Unidos são classificados como cardioembólicos, sendo a fibrilação atrial não valvar responsável por 50\% desses eventos $^{13}$. A incidência anual estimada é de aproximadamente 125.000 casos/ano. A freqüência de AVCi cardioembólicos depende dos critérios utilizados para a definição, do grau de investigação etiológica realizada e do desenho do estudo clínico considerado, variando de 14 a 31\% nas principais séries da literatura ${ }^{16}$. Quanto mais velha a população analisada, maior a freqüência de cardioembolia, uma vez que há um rápido aumento da prevalência de fibrilação atrial com o aumento da idade ${ }^{13,16}$.

Em países como os Estados Unidos, negros e hispânicos parecem ter uma prevalência menor de AVCi cardioembólicos, uma vez que a prevalência de fibrilação atrial nesses grupos raciais é menor. A freqüência relativa do AVCi cardioembólico como uma proporção de todos os AVC é bimodal, sendo maior em pacientes jovens ( $<50$ anos) e mais velhos ( $>75$ anos) $)^{13,16}$. No Brasil e na América do Sul como um todo, a doença de Chagas, causada pelo Trypanosoma cruzi, é uma importante causa de miocardiopatia e AVCi, afetando 16 a 18 milhões de pessoas ${ }^{17}$.

\section{Definição}

Pelos critérios TOAST (Trial of Org 10172) de classificação etiológica dos AVCi, um infarto cerebral é considerado cardioembólico quando oclusões arteriais intracranianas decorrem de um êmbolo que teve origem cardíaca. ${ }^{18}$ As possíveis fontes emboligênicas cardíacas são divididas em alto risco e médio risco, dependendo de suas propensões relativas à embolia (Tabela 1). Pelo menos uma fonte cardíaca emboligênica deve ser encontrada para o diagnóstico de AVCi cardioembólico possível ou provável. Evidência de AVCi prévios em mais de um território vascular ou a presença de embolia sistêmica fortalecem o diagnóstico de AVCi cardiogênico ${ }^{18}$.

\section{Coexistência de AVCi e Doença Coronariana}

O prognóstico precoce e tardio de pacientes com AVCi é criticamente influenciado pela coexistência de doença coronariana. Infarto agudo do miocárdio (IAM) e morte súbita são as principais causas de óbitos em pacientes com doença cerebrovascular a longo prazo $^{19}$. Um terço dos pacientes com AIT, AVCi ou doença carotídea assintomática tem história de IAM ou angina. Pacientes com doença cerebrovascular e doença carotídea têm um risco significativo de eventos coronarianos a longo prazo. Após um AIT ou AVCi, o risco de IAM ou morte por doença coronariana é 2 a 2,5 vezes o da recorrência de AVCi ou morte por doença cerebrovascular ${ }^{20,21}$. Esses dados demonstram que uma avaliação cardiológica de rotina em todos os
Tabela 1. Classificação TOAST* das fontes cardioembólicas: alto e médio risco.
Alto Risco
Válvulas prostéticas mecânicas
Estenose mitral com fibrilação atrial
Trombo em átrio esquerdo
Doença do nó sinusal
Infarto agudo do miocárdio recente ( $<4$ semanas)
Trombo em ventrículo esquerdo
Miocardiopatia dilatada
Acinesia segmentar em ventrículo esquerdo
Mixoma atrial
Endocardite infeciosa

\section{Médio Risco}

Prolapso de válvula mitral

Calcificação de ânulo mitral

Estenose mitral sem fibrilação atrial

Contraste espontâneo em átrio esquerdo

Aneurisma de septo atrial

Forame oval patente

Flutter atrial

Fibrilação atrial isolada

Válvula cardíaca biológica

Endocardite bacteriana não infecciosa

Insuficiência cardíaca congestiva

Segmento ventricular hipocinético

Infarto do miocárdio ( $>4$ semanas, $<6$ meses)

*Trial of Org 10172 in Acute Ischemic Stroke.

pacientes com doença cerebrovascular pode ajudar não só a identificar fontes cardíacas de embolia, como também a selecionar doentes de alto risco para doença coronariana que podem se beneficiar de tratamentos mais agressivos.

\section{Trombólise e AVCi Cardioembólico: entendendo as peculiaridades}

\section{Trombólise e subtipos de AVCi}

Em 1996, o FDA (Food and drug administration) aprovou o uso do rt-PA para o tratamento do AVCi quando administrado até 3 horas do início dos sintomas ${ }^{22}$. O primeiro consenso brasileiro do tratamento da fase aguda do AVC, publicado em 2001, também recomenda o tratamento com rt-PA até três horas do início do quadro neurológico em pacientes com AVCi²3. A aprovação desse tratamento se baseou principalmente na publicação do estudo NINDS (National Institute of Neurological Disorders and Stroke), que demonstrou significativa melhora no prognóstico neurológico em três meses nos pacientes tratados com rt-PA intravenoso dentro de três horas do início dos sintomas. Um prognóstico favorável nesse estudo foi descrito para todos os subtipos de AVCi22. No entanto, no estudo NINDS os subtipos etiológicos de AVCi foram determinados com base na apresentação clínica e em poucos testes diagnósticos.

Um estudo posterior ao NINDS analisou de maneira retrospectiva uma série de 40 pacientes submetidos à trombólise intravenosa com rt-PA e classificados quanto 
à etiologia segundo os critérios TOAST após a realização de uma série de testes diagnósticos. Nesse estudo, 48\% dos AVCs foram cardioembólicos e não houve diferença no prognóstico dos pacientes tratados com rt-PA entre os diferentes subgrupos etiológicos ${ }^{24}$.

\section{Tempo de Recanalização Arterial Espontânea e Transformação Hemorrágica em AVCi Cardioembólico}

A correlação entre tempo de reperfusão e transformação hemorrágica é bem demonstrada em modelos animais de $\mathrm{AVCi}$, ocorrendo mais freqüentemente quando a reperfusão do tecido isquêmico ocorre com mais de três horas do início do processo ${ }^{6}$. Em humanos, um estudo utilizando Doppler transcraniano (DTC) para detectar o tempo de reperfusão em pacientes com fibrilação atrial e AVCi em território da ACM com até seis horas do início dos sintomas demonstrou que recanalização tardia (> seis horas do início dos sintomas) se associa a um maior risco de transformação hemorrágica de forma independente ${ }^{25}$. Outros fatores associados à transformação hemorrágica foram: pontuação na escala de AVC do NIH (National Institute of Health) >14, oclusão proximal da ACM e hipodensidade de mais que $>33 \%$ do território da ACM. Portanto, uma recanalização precoce parece ser um fator fundamental não só na parada da progressão da lesão isquêmica como também na prevenção de transformações hemorrágicas ${ }^{25}$.

\section{Recanalização arterial em pacientes com AVCi cardioembólico tratados com rt-PA}

A base fisiológica do uso do rt-PA no tratamento do AVCi é a capacidade desta medicação de recanalizar artérias ocluídas, salvando, assim, o tecido cerebral da injúria isquêmica ${ }^{26}$. Apesar do tratamento com rt-PA intravenoso ser efetivo em pacientes com AVCi de todos os subtipos, a resposta à medicação pode variar dependendo do tamanho, da composição e da fonte do trombo ou êmbolo². Alguns pacientes de alto risco para trombos cardíacos (como pacientes com infarto agudo do miocárdio recente) foram excluídos dos principais estudos multicêntricos de rt-PA intravenoso no tratamento do AVCi22,28,29. Aspectos estruturais dos trombos têm recebido atenção no que diz respeito à susceptibilidade à penetração dos agentes trombolíticos ${ }^{2}$. Trombos mais velhos, bem organizados e ricos em plaquetas parecem ser mais resistentes à trombólise do que trombos frescos, ricos em fibrina e hemácias, formados em condições de estase sangüínea. A estrutura física, a bioquímica e a composição celular do êmbolo cerebral parecem ser diferentes dependendo da fonte emboligênica. Por exemplo, um trombo aderido a uma lesão aterosclerótica proximal tem sua composição diferente do formado em cavidades cardíacas ${ }^{30,31}$.

A recanalização é um processo contínuo que geralmente se inicia precocemente após a administração do rt-PA. No entanto, o tempo para completa dissolução do trombo e restauração do fluxo sangüíneo depende da localização da oclusão, da composição do trombo, da área de superfície do trombo exposta ao fluxo sangüíneo e da pressão que permite a penetração do rt-PA na estrutura do trombo ${ }^{32}$.

Um estudo analisou a relação entre recanalização arterial detectada através do Doppler transcraniano (DTC), volume do infarto e prognóstico neurológico, em pacientes com AVCi cardioembólico tratados com rt-PA intravenoso. A taxa de recanalização em seis horas detectada através do DTC foi maior no grupo tratado com rt-PA intravenoso (65\%) do que no grupo controle (15\%). O volume do infarto foi significativamente menor no grupo tratado com rt-PA $\left(50,2 \pm 40,3 \mathrm{~cm}^{3}\right)$ do que no grupo controle $(124,8 \pm 81,6$ $\left.\mathrm{cm}^{3}\right)$. Em três meses, $58 \%$ dos pacientes submetidos à trombólise estavam independentes funcionalmente (escala modificada de Rankin <=2), comparados a apenas 23\% dos controles. O prognóstico clínico se correlacionou fortemente com o grau de recanalização em seis horas. Nesse estudo, uma regressão logística mostrou a escala de AVC do $\mathrm{NIH}<17$ e a recanalização precoce (até seis horas ) como fatores independentes no prognóstico funcional em três meses ${ }^{33}$.

\section{Diferentes taxas de recanalização em diferentes subtipos de AVCi}

Uma vez demonstrada a maior taxa de recanalização arterial em pacientes com AVCi cardioembólicos tratados com rt-PA quando comparados a pacientes que não receberam essa medicação, restava a dúvida se havia diferenças nas taxas de recanalização arterial entre os diferentes subtipos de AVCi ${ }^{33}$.

Um estudo foi então desenhado para comparar as taxas de recanalização arterial (detectada através de DTC) em pacientes com oclusão da ACM tratados com rt-PA intravenoso entre os diferentes subtipos de AVCi segundo a classificação do estudo TOAST. Durante a primeira hora de infusão do rt-PA, recanalização arterial ocorreu em $47 \%$ dos pacientes, sendo súbita em $32 \%$, progressiva (melhora das velocidades de fluxo sangüíneo em 1 a 29 minutos) em 50\% e lenta (melhora das velocidades de fluxo sangüíneo em 30 minutos ou mais) em $18 \%$ dos pacientes. Recanalização em uma hora foi mais freqüente em pacientes com AVCi cardioembólico (59\%) , quando comparada à recanalização em pacientes com doença de grandes artérias (8\%) e com aqueles com AVCi de etiologia indeterminada (50\%). Uma fonte cardíaca emboligênica foi diagnosticada em $81 \%$ dos pacientes com recanalização arterial súbita durante a infusão do rtPA. A recanalização súbita se correlacionou com melhora neurológica em 24 horas, quando comparada à recanalização progressiva e à lenta. Com esse trabalho, ficou bem demonstrado que as taxas de recanalização variam entre os diferentes subtipos de AVCi e que pacientes com AVCi cardioembólico apresentam com maior freqüência recanalização completa e precoce ${ }^{34}$. 


\section{Trombólise Intra-arterial e AVCi Cardioembólico}

Apesar dos resultados citados anteriormente, que definem que pacientes com AVCi cardioembólico têm uma maior taxa de recanalização quando tratados com rt-pA intravenoso se comparados a pacientes com AVCi aterotrombótico de grande artérias, muitas vezes o tratamento com trombolítico intravenoso não pode ser utilizado por limitações de tempo (mais de três horas do início dos sintomas) ou por uso prévio de medicações anticoagulantes. As taxas de recanalização citadas em AVCi cardioembólicos parecem ser reais para pacientes com AVCi cardioembólicos secundários a fibrilação atrial em que êmbolos podem ser recém-formados e vermelhos (ricos em fibrina e eritrócitos) ${ }^{33}$. Trombos de origem ventricular, muitas vezes com altos teores de cálcio, ou mesmo os originados em pacientes com doença valvar, podem não responder tão bem à trombólise intravenosa ${ }^{10}$.

A trombólise intra-arterial parece ser uma opção para o tratamento de pacientes com AVCi cardieombólico que não podem ser tratados com rt-PA intravenoso. As taxas de recanalização em pacientes com oclusão proximal da ACM são maiores em pacientes tratados com trombólise intra-arterial (70\%), quando comparados àqueles tratados com trombolítico intravenoso $(31 \%)^{33,35}$. A menor dose do agente trombolítico utilizado na trombólise intra-arterial a torna mais segura em pacientes com maior risco de sangramento (como em pacientes em pós-operório recente de cirugia cardíaca ou naqueles tratados com anticoagulantes orais).

O estudo PROACT II, que tratou com pro-uroquinase intra-arterial pacientes com oclusões de ACM (M1 e/ou M2), demonstrou que esse procedimento pode melhorar o prognóstico de pacientes tratados dentro de seis horas do início dos sintomas ${ }^{35}$. Nesse estudo, o número de pacientes tratados necessário para evitar um caso de morte ou dependência foi sete. A alta taxa de hemorragia intracraniana sintomática no estudo PROACTII (10\%), quando comparada às taxas de hemorragia nos estudos de rt-PA intravenoso (6,4\% (NINDS), 8,8\% (ECASSII) e 7,2\% (ATLANTIS)) levou à rejeição pelo FDA dessa medicação $22,28,29,35$. No entanto, com base no fato de que as maiores taxas de sangramento nesse estudo podem ter decorrido da gravidade do AVCi na população tratada (NIH médio de 17, comparado a 11 no ECASS II e ATLANTIS e 14 no NINDS), ao maior tempo para o tratamento e à maior taxa de recanalização atingida, a trombólise intra-arterial continua a ser utilizada ${ }^{35}$. Em nosso país e nos Estados Unidos, a medicação trombolítica usada em procedimentos de trombólise intra-arterial tem sido o rt-PA, uma vez que não disposmos de pro-uroquinase.

Uma outra aplicação importante da trombólise intraarterial em pacientes com doença coronariana é a sua realização em AVCi embólicos durante procedimentos de cinecoronariografia. Um estudo restrospectivo envolvendo três hospitais universitários mostrou que, em 21 pacientes tratados com trombólise intra-arterial por AVCi embólicos peri-procedimento angiográfico, este tratamento foi seguro e não aumentou o risco de hemorragia intracraniana sintomática ou de morte $^{36}$.

\section{Caso Clínico llustrativo}

Paciente do sexo feminino, 57 anos, com história prévia de troca valvar mitral há 20 anos, foi admitida ao hospital São Paulo por ter sido encontrada sonolenta após ter apresentado diversos episódios de náuseas e vômitos. O horário do início dos sintomas era incerto. Evoluiu dentro do hospital com piora do nível de consciência, anartria, hemiplegia direita e desvio do olhar conjugado para a direita (escala do $\mathrm{NIH}=16$ ). Realizou tomografia computadorizada de crânio que não mostrou alterações significativas. Pela incerteza do horário de início dos sintomas e pelo uso prévio de anticoagulante oral, optou-se por submeter a paciente a procedimento de trombólise intra-arterial.

Angiografia cerebral diagnóstica mostrou oclusão da basilar em terço médio. Foi então realizado procedimento endovascular com passagem do cateter pela obstrução, com injeção de 2 mg de rt-PA após obstrução, 2 mg no trombo e posterior infusão contínua do rt-PA, obtendo-se fluxo TIMI 3 (recanalização completa) com a dose total de 8,5mg da medicação (Figura 2). Após o procedimento, a paciente evoluiu com melhora importante do quadro neurológico, permanecendo vigil, com fala fluente e com melhora da força em hemicorpo direito (escala do $\mathrm{NIH}=8$ ).

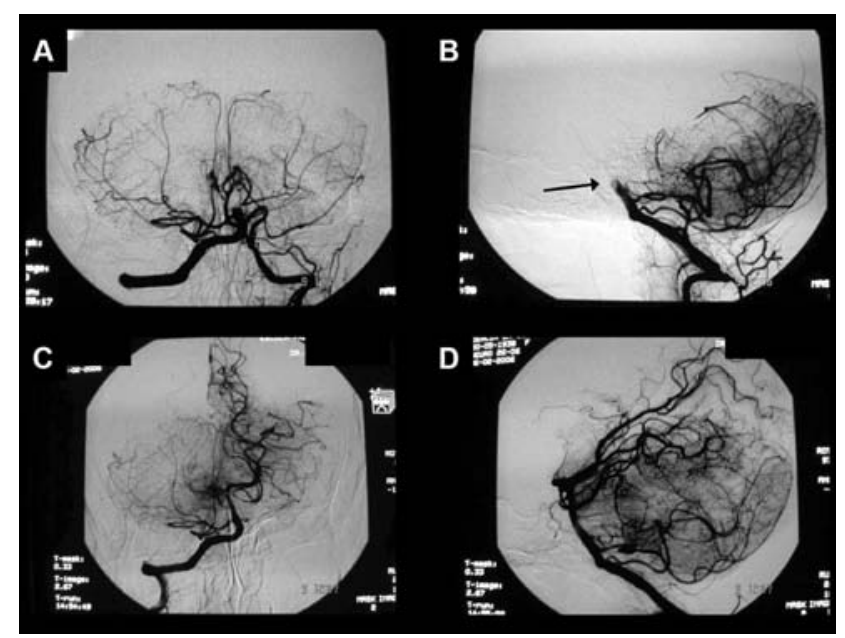

Figura 2:

2 A e B - Angiografia digital mostrando oclusão em terço médio da artéria basilar em paciente com válvula mitral biológica.

2C e D - Recanalização em artéria basilar após injeção intra-arterial de $8,5 \mathrm{mg}$ de $r t-P A$. 


\section{CONCLUSÃO}

O manejo clínico da fase aguda do AVCi cardioembólico segue os princípios gerais do tratamento do AVCi. No entanto, o conhecimento das suas peculiaridades clínicas permite um diagnóstico precoce de cardioembolia e a realização de procedimentos de trombólise com atenção a algumas particularidades como a maior chance de reperfusão e de sangramento e o risco da não recanalização arterial em pacientes com êmbolos contendo cálcio. O tratamento intra-hospitalar após o procedimento trombolítico no $\mathrm{AVCl}$ cardioembólico é fundamental na evolução desses pacientes e deve ser feito por equipes multidisciplinares, sendo a participação do cardiologista integrado à equipe de neurologia de muita importância, uma vez que a estabilização do quadro cardíaco pode evitar a recorrência de eventos embólicos.

\section{Agradecimento}

Agradeço ao Dr. Gustavo Kuster pela documentação das imagens do caso clínico ilustrativo.

\section{REFERÊNCIAS BIBLIOGRÁFICAS}

1. Versalius A. De Humani Corpis Frabica. Basilae, J Oporini, 1543.

2. Virchow R. Uber die akut entzundung der arterien. Virchows Arch Patho Anat 1846; 1:272-378

3. Osler W. The principles of practice of Medicine.5th ed. New York: D. Appleton and Co., 1903:1008-1009.

4. Fisher CM. Occlusion of the internal carotid artery. Arch Neurol 1951; 65:346377.

5. Liebeskind A, Chinichian A, Schechter M. The moving embolus seen during cerebral angiography. Stroke 1971; 2:440-443.

6. Fisher CM, Adams RD. Observations on brain embolism with special reference to the mechanism of hemorrhagic infarction. J Neuropathol Exp Neuro 1951:10: 92-94.

7. Okada Y, Yamaguchi T, Minematsu K, Miyashita T, Sawada T, Sadoshima $\mathrm{S}$, et al. Hemorrhagic transformation in cerebral embolism. Stroke 1989; 20:598-603

8. de Freitas GR, Carruzzo A, Tsiskaridze A, Lobrinus JA, Bogousslavsky J. Massive haemorrhagic transformation in cardioembolic stroke: the role of arterial wall trauma and dissection. J Neurol Neurosurg Psychiatry 2001 70(5): 672-674.

9. Mohr JP, Caplan LR, Melski JW, Goldstein RJ, Duncan GW, Kistler JP, et al The Havard Cooperative Stroke Registry: a prospective registry. Neurology 1978; 28:754-762

10. Timsit SG, Sacco RL, Mohr JP, Foulkes MA, Tatemichi TK, Wolf PA, et al. Brain infarction severity differs according to cardiac or arterial embolic source. Neurology 1993; 43:728-733.

11. Helgason C. Cardioembolic stroke topography and pathogenesis. Cerebrovasc Brain Metab Rev 1992; 4:28-58.

12. Caplan LR, Hier DB, D ' Cruz I. Cerebral embolism in the Michael Reese Stroke Registry. Stroke 1983; 14:530-536

13. Cerebral Embolism Study Group. Cardiogenic brain embolism: The second report of the Cerebral Embolism Task Force. Arch Neurol 1989; 46 : 727-743.

14. Bogousslavsky J, Cachin C, Regli F, Despland PA, Van Melle G, Kappenberger L. Cardiac sources of embolism and cerebral infarction. Clinical consequences and vascular concomitants. Neurology 1991; 41:855-859.

15. Caplan LR. Posterior Circulation Disease. Clinical Findings, Diagnosis and Management. Boston, Blackwell Science, 1996.

16. Grau AJ, Weimar C, Buggle F, Heinrich A, Goertler M, Neumaier S, et al Risk factors, outcome, and treatment in subtypes of ischemic stroke: The German Stroke Data Bank. Stroke 2001; 32: 2559-2566.

17. Carod-Artal FJ, Vargas AP, Melo M, Horan TA. American trypanosomiasis (Chagas `disease): an unrecognized cause of stroke. J Neurol Neurosurg Psychiatry 2003; 74:516-518.

18. Adams HP Jr, Bendixen BH, Kappelle LJ, Biller J, Love BB, Gordon DL, et al. Classification of subtype of acute ischemic stroke. Definitions for use in a multicenter clinical trial. TOAST. Trial of Org10172 in Acute Stroke Treatment. Stroke 1993; 24:35-41.

19. Wilterdink JL, Furie KL, Easton JD. Cardiac evaluation of stroke patients. Neurology 1998; suppl 3:S23-S26.

20. Chimowitz MI, Weiss DG, Cohen SL, Starling MR, Hobson RW 2nd. Cardiac prognosis of patients with carotid stenosis and no history of coronary artery disease. Stroke 1994; 25:759-765.
21. Loh E, Sutton MSJ, Wun CC. Ventricular dysfunction and the risk of stroke after myocardial infarction. N Engl J Med 1997; 336:251-257.

22. The National Institutes of Neurological Disorders, and Stroke rt-PA Stroke Study Group. Tissue plasminogen activator for acute ischemic stroke. N Engl J Med. 1995; 333: 1581-1587

23. Primeiro Consenso Brasileiro do Tratamento da Fase Aguda do Acidente Vascular Cerebral. Arq. Neuro-Psiquiatr 2001; 59 (4):675-680.

24. Hsia AW, Sachdev HS, Tomlinson J, Hamilton SA, Tong DC. Efficacy of IV tissue plasminogen activator in acute stroke: Does stroke subtype really matter? Neurology 2003. 61(1): 71-75

25. Molina CA, Montaner J, Abilleira S, Ibarra B, Romero F, Arenillas JF, et al Timing of spontaneous recanalization and risk of hemorrhagic transformation in acute cardioembolic stroke. Stroke. 2001; 32: 1079-1084.

26. Overgaard K, Sereghy T, Boysen G, Pedersen H, Diemer NH. Reduction of infarct volume and mortality by thrombolysis in a rat embolic stroke model. Stroke. 1992; 23: 1167-1173.

27. Zhang RL, Chopp M, Zhang ZG, Divine G. Early (1h) administration of tissue plasminogen activator reduces infarct volume without increasing hemorrhagic transformation after focal cerebral embolization in rats. J Neurol Sci. 1998; 160: 1-8.

28. Hacke W, Kaste M, Fieschi C, von Kummer R, Davalos A, Meier D, et al. Randomised double-blind placebo-controlled trial of thrombolytic therapy with intravenous alteplase in acute ischemic stroke (ECASS II) Lancet. 1998 352: 1245-1251.

29. Clark WM, Wissman S, Albers GW, Jhamandas JH, Madden KP, Hamilton S. Recombinant tissue-type plasminogen activator (alteplase) for ischemic stroke 3 to 5 hours after symptom onset: the ATLANTIS study: a randomized controlled trial: Alteplase Thrombolysis for Acute Noninterventional Therapy in Ischemic Stroke. JAMA 1999: 282: 2019-2026.

30. Fagan SC, Garcia JH. Hemorrhagic transformation in focal cerebral ischemia: influence of time to artery reopening and tissue plasminogen activator Pharmacotherapy. 1999; 19: 139-142.

31. Caplan LR, Mohr JP, Kistler JP, Koroshetz W. Should thrombolytic therapy be the first-line treatment for acute stroke? Thrombolysis: not a panacea for ischemic stroke. N Engl J Med. 1997; 337: 1309-1310.

32. Christou I, Alexandrov AV, Burgin WS, Wojner AW, Felberg RA, Malkoff M et al. Timing of recanalization after tissue plasminogen activator therapy determined by transcranial Doppler correlates with clinical recovery from ischemic stroke. Stroke. 2000; 31: 1812-1816.

33. Molina CA, Montaner J, Abilleira S, Arenillas JF, Ribo M, Huertas R, et al Time course of tissue plasminogen activator-induced recanalization in acute cardioembolic stroke: a case-control study. Stroke. 2001; 32: 2821-2827.

34. Molina CA, Montaner J, Arenillas JF, Ribo M, Rubiera M, Alvarez-Sabin J. Differential Pattern of Tissue Plasminogen Activator-Induced Proximal Middle Cerebral Artery Recanalization Among Stroke Subtypes. Stroke 2004; 35(2): 486-490.

35. Furlan A, Higashida R, Wechsler L, Gent M, Rowley H, Kase C, et al. Intraarterial prourokinase for acute ischemic stroke. The PROACT II study: A randomized controlled trial. Prolyse in Acute Cerebral Thromboembolism. JAMA 1999:282:2003-2011.

36. Zaidat OO, Slivka AP, Mohammad Y, Graffagnino C, Smith TP, Enterline DS, et al. Intra-Arterial Thrombolytic Therapy in Peri-Coronary Angiography schemic Stroke Stroke 2005: 36:1089-1090. 